\title{
In Situ TEM Study of the Hydrogen Effect on the Interface between Al and Its Oxide at Room and Elevated Temperature
}

\author{
Meng $\mathrm{Li}^{1}$, Degang Xie ${ }^{1}$, Zhiwei Shan ${ }^{1}$ \\ ${ }^{1 .}$ Center for Advancing Materials Performance from the Nanoscale (CAMP-Nano), State Key \\ Laboratory for Mechanical Behavior of Materials, Xi'an Jiaotong University, Xi'an, China.
}

Fossil fuels, including coal, natural gas and petroleum, are the major energy source for most of the countries. However, the consumption of fossil fuels is expected to generate many kinds of emissions, such as carbon dioxide, carbon monoxide, nitrogen dioxide, sulphur dioxides and industrial dust etc. Once the total amount of these emissions exceeds the upper limit of environmental capacity, heavy air pollution such as haze will occur which has raised serious concerns regarding its potential threat not only on human health, but also on precision machinery and high cleanliness industry [1]. In order to satisfy the global rising energy demands, intense research efforts have been dedicated worldwide to seek other renewable, clean and green energy sources, such as atomic power, solar energy, wind energy and hydrogen energy etc. Among them, hydrogen energy is thought to be promising because it is non-toxic, renewable, bountiful in supply and far more efficient than other sources of energy. However, to make hydrogen energy practically affordable and user friendly, people need to generate, store and transport hydrogen in a safe and low-cost manner.

Despite of its admirable benefits as a green energy source, hydrogen is notorious in deteriorating the mechanical properties of materials. For example, hydrogen can embrittle the materials, foster the nucleation and growth of cavitation/blistering and undermine the integrity of the interface between base materials and their protection surface layer. In addition, such hydrogen-induced damage is known to be exacerbated at elevated temperature, which is frequently seen under $\mathrm{H}$-containing environment in gas turbines, power plants, petrochemical factories, and solar sails. Passivating oxide layer, in particular that of chromium or aluminum, is often applied to improve the corrosion resistance of metals. However, the integrity of this layer is usually sabotaged by moisture, especially upon hydrogen attack at elevated temperatures. In order to minimize or eliminate the aforementioned hydrogen damage, it is necessary to understand their physical origin which remain elusive so far, especially at the atomic scale.

Using in situ environmental transmission electron microscopy, we demonstrated that once the atomic bonding at the aluminum metal/oxide interface is severed by the segregating hydrogen, surface-diffusion of $\mathrm{Al}$ atoms sets in to initiate facet-forming shape changes as dictated by Wulff construction, producing numerous gas-accumulating cavities at the metal side of the metal/oxide interface. The surface oxide layer remains unyielding until the metal-side cavities grow to a critical size above which the accumulated gas pressure become strong enough to push out and blister the oxide layer. The rate of this diffusional deformation is sensitively dependent on the crystallographic orientation of the Al [2]. Further, by heating up hydrogenated aluminum samples, we found that there exists a critical temperature of $\sim 150{ }^{\circ} \mathrm{C}$, above which the growth of cavities at the metal-oxide interface reverses to shrinkage, followed by the formation of a few giant cavities. Vacancy super-saturation, activation of a long-range diffusion pathway along the detached interface and the dissociation of hydrogen-vacancy complexes are critical factors affecting this behavior [3]. 
Our findings highlight the important role of metal surface diffusion in setting up gas-accumulating pockets and the subsequent formation of giant cavity, as such have broad implications for designing new strategies to improve coating performance. Our observation also opens up a promising route to create novel quasi2D materials, by purposely exfoliating ultrathin oxide films via exposure to hydrogen [4].

\section{References:}

[1] M. Ding, W. Han, J. Li, E. Ma, Z. Shan, Science China Technological Sciences (2015).

[2] D. Xie, et al, Nat. Mater., 14 (2015), p. 899.

[3] M. Li et al, Nature Communications, 10.1038/NCOMMS14564 (2017).

[4] The authors acknowledge the support from the National Natural Science Foundation of China (51231005 and 51621063)
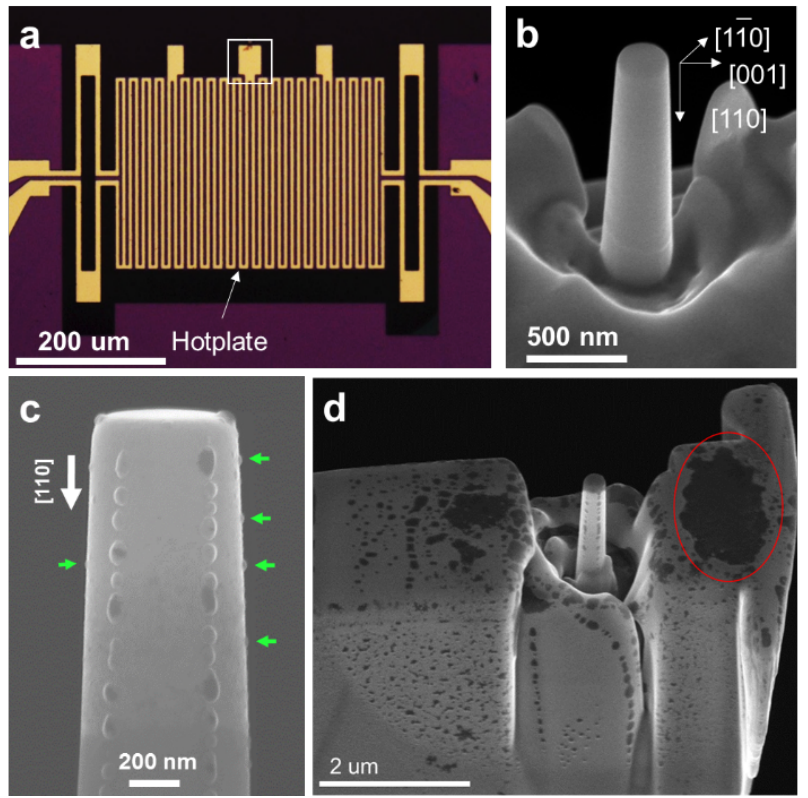

Figure 1. (a) Illustration of a home-made MEMS heating chip with precise temperature sensing availability. (b) aluminum pillar sample before hydrogenation. (c) Crystal orientation dependent hydrogen blisters formed at room temperature. (d) after heating treatment, numerous cavities (dark contrast) form under the surface layer, note the giant cavity framed by the red ellipse. 